\title{
Content Based Image Retrieval: Bone Age Assessment
}

\author{
Manjula Gururaj $\mathbf{H}^{1}$, Dr. G. S. Nagaraja ${ }^{2}$ \\ ${ }^{1}$ Research Scholar, JAIN University, Bengaluru, India \\ ${ }^{2}$ Professor, Computer Science \& Engineering, R V College of Engineering, Bengaluru, India
}

\begin{abstract}
Bone age assessment using a hand radiograph is an important clinical tool in the area of paediatrics, especially in relation to endocrinological problems and growth disorders. This paper proposes Standard Deviation based skeletal Bone Age Assessment based on features extracted from phalanx. The system works according to the renowned Tanner and Whitehouse method based on the Phalanx Region of Interest. The system ensures accurate and robust bone age assessment of children between the age group 0-10 years. The proposed work focuses on pre-processing stage in which cropping the phalanx ROI from the input image by followed by filter is done. This follows morphological feature extraction during which three geometric features are extracted, one from each of the phalanx leading to a set of 14 features. The extracted features are used to train the system in the beginning till end to estimate the bone age. The system is tested on a set of radiographs and the results are discussed.
\end{abstract}

Keywords: Carpal region, Region of Interest, Standard deviation method, Euclidean distance

\section{Introduction}

Bone age assessment is a procedure frequently performed in paediatric radiology. Based on radiological examination of skeletal development of hand wrist, bone age is assessed and then compared with chronological age. A discrepancy between these two values indicates abnormalities in skeletal development. The procedure is often used in the management and diagnosis of endocrine disorders and it can also serve as an indication of the therapeutic effect of treatment. Generally, it can indicate whether the growth of a patient is accelerating or decreasing. In many cases the decision whether to treat a patient with growth hormones depends on the outcome of this test. Another relevant application is in the social field. Determination of the skeletal maturity can help in the determination of the true age of such a person. This examination is universally used due to its simplicity, minimal radiation exposure, and the availability of multiple ossification centres for evaluation of maturity.

The main clinical methods for skeletal bone age evaluation are the Greulich and pyle(GP) method and the Tanner E Whitehouse(TW2) method. Automatic skeletal age assessment has the potential to reduce the time required to examine the image and to increase the reliability of the analysis. There are several differences between two methods. The GP method is most widely used method and is faster and easier to use than TW2 method. These two methods produce different values for skeletal age and these differences are significant in clinical practice.Tw2 method is more reproducible of the two, and also potentially more accurate.

In GP method, large number of children, wrist radiograph images of different ages is enrolled in a database. These images are examined, and data compared, with the "Atlas of Skeletal Maturation of the Hand" to find the age. This method is used to find the more details of individual bones and epiphyses.
In TW2 method, 20 regions of interest (ROIs) located in the main bones are considered for bone age evaluation. Each ROI is divided into three parts: epiphysis, metaphysis and diaphysis as shown in the Fig. 1.

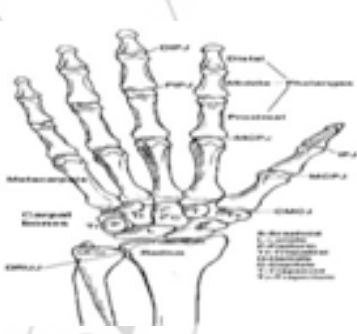

Figure 1: Parts of the wrist

Computerized Convex Hull Method of Skeletal Bone Age Assessment from Carpal Bones [1] used to find the age of the radiographic images. Here, the system works according to the renowned TW2 method, based on the Carpal Region of Interest (CROI). The system ensures accurate and robust BAA for the age range 0-10 years for both girls and boys. Pre-processing consists of initially cropping the CROI from the input image, followed by applying anisotropic diffusion filter. This is followed by morphological feature extraction during which three geometric features are extracted, one from each of the 7 carpal bones, thus leading to a feature set of 21 features. The extracted features are used to train the system since beginning til end to estimate the bone age.

The development of each ROI is divided into discrete stages and a numerical score is associated with each bone and by adding the scores of all ROIs, an overall maturity score is obtained. With the exponential increase in the size of digital image databases in the past few years, traditional way of manually annotating the images with text and then using textbased queries for image retrieval has been giving way to Content-based Image Retrieval (CBIR) Systems that use the visual contents of the images to automatically index and retrieve digital images. However, there is always a gap between high-level human perception of an image and the low-level image features used to describe its contents. This 


\section{International Journal of Science and Research (IJSR) \\ ISSN (Online): 2319-7064}

Index Copernicus Value (2013): 6.14 | Impact Factor (2015): 6.391

gap between low-level image features and semantic image content is the major bottleneck faced by traditional CBIR systems. Modern CBIR systems overcome this problem by using interactive learning, bringing the user in the loop. Such systems learn from feedbacks given by the user.

\section{Proposed Approach}

The proposed work make use of Content-based image retrieval (CBIR), which promises comparisons of the images and regions of interest (ROI) to earlier cases without the need to specify many separate heuristics. Different approaches have been taken to fully or partially automate bone age assessment. In order to assess the bone age for a given hand radiograph, the epiphysis regions (eROIs) between metacarpals and are compared to the corresponding regions of all hand radiographs with known age in the retrieval database. From these similarities the unknown age of the radiographic image is calculated. If the similar images are not present then the bone age is calculated depending on the standard deviation, Euclidean method which is obtained from the phalanges region of the image.

The age assessment for a new radiograph by CBIR is then performed using our algorithm, which consists of four stages, which are:

- The image is first preprocessed.

- 14 eROIs are extracted from the new image.

- For each of the eROI extracted, control points are taken.

- Then using those control points standard deviation methods bone age is predicted.

\section{Modules of the Proposed Approach}

This section discusses different modules of the proposed approach.

1) Image Pre-Processing

2) Image comparison

3) Feature extraction

4) Bone age estimation - which is done through 2 methods: Standard deviation, Euclidean method.

5) Updating, or Feedback

\section{Query Image}

This module takes an input image from the user; the user gives the right hand wrist $\mathrm{X}$-ray image as the input.
Input Radiograph

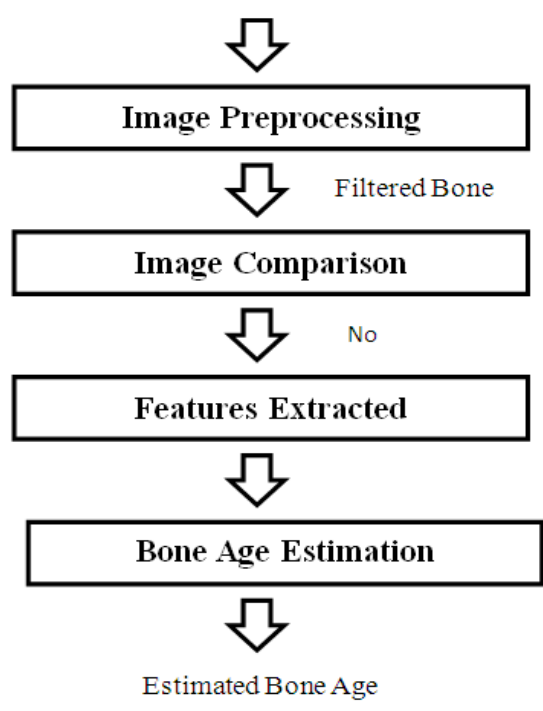

Figure 2: Overall operation

Then the image is pre-processed, the pre-processed image is filtered for clarity, then the images are compared with the stored images. If the image is present the age is retrieved, else features are extracted and the bone age is estimated.

\section{a) Pre-processing of Image}

From the input image, the CROI are cropped initially. The cropped CROI image is pre-processed using anisotropic diffusion filter. The pre-processed image is given as input to the next step that is image comparison.

Image pre-processing is performed in two steps,

- Image smoothing and

- Greyscale conversion.

Image smoothing is done to reduce the noise within the image or to produce a less pixilated image. Most smoothing methods are based on low pass filters. The proposed work uses smoothing to reduce noise by using a Gaussian filter. Gaussian filter reduces the magnitude of higher frequencies proportional to the lower frequencies, but at the cost of more computation time. But the speeding up of smoothing is achieved by splitting 2D Gaussian $\mathrm{G}(\mathrm{x}$, y) into two 1D Gaussians $\mathrm{G}(\mathrm{x}) \mathrm{G}(\mathrm{y})$ and carrying out filtering in $1 \mathrm{D}$, first row by row and then column by column.

Greyscale conversion is done as follows. Colours in an image are converted to a shade of gray by calculating the effective brightness or luminance of the colour and using this to create a shade of gray that matches the desired brightness.

In image pre-processing, the input image is cropped and using canny edge detection, edges in the bones are identified. Med filters can also be used to remove noises.

\section{b) Image Comparison}

After image pre-processing next step is to compare the images. In image comparison first the image is given as input, then the difference of that two images are taken, threshold is found. If the image matches it gives output as same, if the image does not match it gives the output as 


\section{International Journal of Science and Research (IJSR) \\ ISSN (Online): 2319-7064 \\ Index Copernicus Value (2013): 6.14 | Impact Factor (2015): 6.391}

different. Here if the image is already present in the data base, then age present in the database is directly extracted. Otherwise, following step is followed.

\section{c) Bone age Estimation}

Bone age can be computed using two methods as follows.

- Euclidean distance

- Standard Deviation

\section{Euclidean Distance}

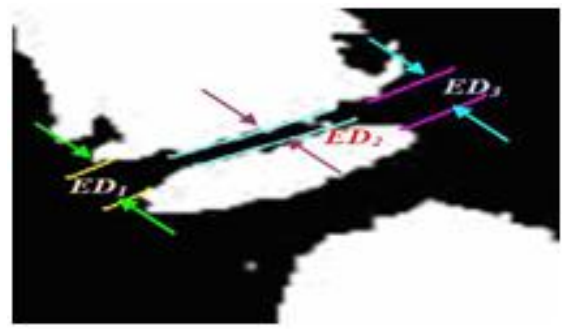

Figure 3: Euclidian distance

In Euclidean distance, distance between the phalanx region is computed like ED1, ED2 and ED3, and then mean of that is computed and substituted in the formula.

Euclidean distance is calculated using control point values.

These contains $\mathrm{X}$ and $\mathrm{Y}$ values of Region selected for age estimation.

\section{d) Standard Deviation method}

In Standard deviation method, age is found based on computing the distances between the phalanxes. Then, mean of the distances is computed and used in the formula to find the age. Control points are used to find the distances, $x$ and $y$ values of the regions are selected for finding the age value. In this work, standard deviations of y values are considered.

\section{e) Updating or Feedback}

Step1: After finding age i.e using the methods standard deviation, and Euclidian distance method for new image. Step 2: The estimated age of image is updated in database.

\section{Results}

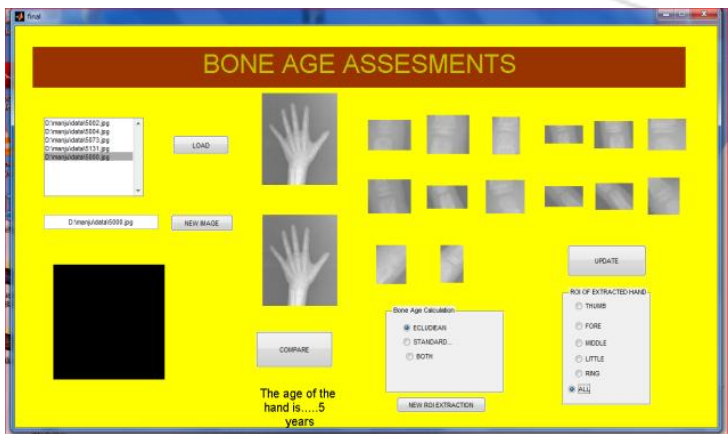

Figure 4: The entire bone age assesment
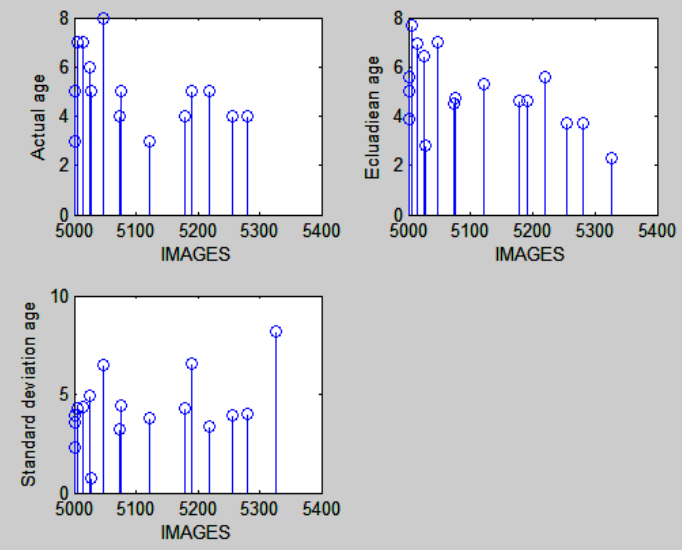

Figure 5: a) Actual age of the images b) The estimated Euclidian age c) The Standard Deviation age

\section{Conclusion}

The proposed Bone Age Assessment System using Euclidean Distance and Standard Deviation method is good analytical method to find the age of children. In the Euclidean distance is very useful that it requires only small amount of data for training and from the results it is evident that the performance of the system is excellent. The adopted approach is found reasonably appropriate for the age group of $0-10$ years since the main finger bones that contribute toward BAA during that stage, which are the Phalanx bones are considered here. Future work would be enhancing this approach on the age group above 10 years, broadening the system to include the further TW2 bones such as radius, ulna, etc. and integrating the system with the clinical PACS.

\section{Acknowledgment}

I am grateful to the valuable guidance provided by Dr. Nagaraja G.S Professor, Department of Computer Science \& Engg, R V College of Engineering, and Bangalore for providing me a valuable help and support and I thank Mrs. Rashmi G, Associate Professor, NITMS, for providing her support.

\section{References}

[1] P. Thangam , K. Thanushkodi , T. V. Mahendiran "Computerized Convex Hull Method of Skeletal Bone AgeAssessment from Carpal Bones", ISSN 1450-216X Vol.70 No.3 (2012) European Journal of Scientific Research.

[2] Skeletal Bone Age Assessment ConcettoSpampinato University Of Catania - Department Of Informatic And Telecommunication Engineering University Of Catania Iale Andrea Doria, 695125 Catania - Italy Cspampin@Diit.Unict.It

[3] J.M. Tanner, M.J.R. Healy, H. Goldstein, N. CameronAssessment of skeletal maturity and prediction of adult height (TW3 method)WB Saunders, London (2001)

[4] E. Pietka, A. Gertych, S. Pospiech, Fei Cao, H. K. Huang and V. Gilsanz, "Computer-assisted bone age assessment: image preprocessing and epiphyseal/metaphyseal ROI 


\section{International Journal of Science and Research (IJSR) \\ ISSN (Online): 2319-7064}

Index Copernicus Value (2013): 6.14 | Impact Factor (2015): 6.391

extraction," in IEEE Transactions on Medical Imaging, vol. 20, no. 8, pp. 715-729, Aug. 2001.

[5] Gertych A, Zhang A, Sayre J, Pospiech-Kurkowska S, Huang H. Bone Age Assessment of Children using a Digital Hand Atlas. Computerized medical imaging and graphics: the official journal of the Computerized Medical Imaging Society. 2007;31(4-5):322-331.

[6] Bull R, Edwards P, Kemp P, Fry S, Hughes I. "Bone age assessment: a large scale comparison of the Greulich and Pyle, and Tanner and Whitehouse (TW2) methods", Archives of Disease in Childhood. 1999;81(2):172-173.

[7] P. Thangam, K. Thanushkodi, T.V. Mahendiran, "PSO for Graph-Based Segmentation of Wrist Bones 2in Bone Age Assessment", INT J COMPUT COMMUN, ISSN 1841-98368(1):153-160, February, 2013.

[8] MEDICAL IMAGE PROCESSINGK.M.M. Rao, V.D.P. Rao

[9] Manzoor Mughal A, Hassan N, Ahmed A.Bone Age Assessment Methods: A Critical Review. Pakistan Journal of Medical Sciences. 2014;30(1):211-215. doi:10.12669/pjms.301.4295. 